Supplemental Material

\title{
Sunlight Induced Photochemical Decay of Oxidants in Natural Waters: Implications in Ballast Water Treatment
}

\author{
William J. Cooper* ${ }^{1}$ \\ Adam C. Jones ${ }^{2}$ \\ Robert F. Whitehead ${ }^{2}$ \\ Rod G. Zika ${ }^{3}$ \\ ${ }^{1}$ Department of Civil and Environmental Engineering \\ University of California, Irvine \\ Irvine, CA 92697-2175 \\ wcooper@uci.edu \\ ${ }^{2}$ Department of Chemistry and Biochemistry, and \\ Center for Marine Science \\ University of North Carolina Wilmington \\ 5600 Marvin K. Moss Lane \\ Wilmington, NC 28409 \\ acjones@ucsd.edu \\ whiteheadrf@uncw.edu \\ ${ }^{3}$ Marine and Atmospheric Chemistry \\ Rosenstiel School of Marine and Atmospheric Sciences \\ University of Miami \\ 4600 Rickenbacker Causeway \\ Miami, FL 33149 \\ rzika@rsmas.miami.edu \\ Environmental Science and Technology
}

February 15, 2007

Supplementary information contains 27 pages, 1 figure, 5 tables and 33 references. 


\section{Overview of the photochemical decomposition mechanisms of hypohalous acids and $\mathrm{ClO}_{2}$ in aqueous solution.}

The photochemical mechanisms of several of the oxidants under consideration for ballast water treatment have been extensively studied under controlled experimental conditions in the laboratory. For example, the primary reaction products generated in the near ultraviolet for alkaline solutions of hypochlorite were $\mathrm{O} \bullet, \mathrm{O}^{-}, \mathrm{Cl} \bullet$, and $\mathrm{Cl}^{-}(1-6)$. Proposed intermediate reaction products included $\mathrm{ClO}_{2}{ }^{-}, \mathrm{Cl}_{2} \mathrm{O}_{2}$ and $\mathrm{ClO} \bullet$ all of which lead, through a series of reactions, to the final products, $\mathrm{Cl}^{-}, \mathrm{ClO}_{3}{ }^{-}$and $\mathrm{O}_{2}(3)$. The photochemistry of $\mathrm{OBr}^{-}$in the near-ultraviolet was very similar to hypochlorite (5-7), with the primary products being $\mathrm{O}^{\bullet}, \mathrm{O}^{-}, \mathrm{Br}^{\bullet}$, and $\mathrm{Br}^{-}$. $\mathrm{The}^{-}$ intermediate products proposed were $\mathrm{BrO}_{2}^{-}, \mathrm{Br}_{2} \mathrm{O}_{2}, \mathrm{BrO} \cdot, \mathrm{Br}_{2} \mathrm{O}_{4}$, and lead to the final products $\mathrm{Br}^{-}, \mathrm{BrO}_{3}{ }^{-}$and $\mathrm{O}_{2}(2,8-12)$. Sunlight exposure of chlorinated seawater, which contains $\mathrm{HOBr} / \mathrm{OBr}^{-}$, yields bromate ion as a major product (13-15). For both chlorine and bromine solutions, the formation of strong oxidizing radicals during photolysis can lead to a variety of intermediate halogen free radical species:

$$
\begin{aligned}
& \mathrm{HO} \bullet+\mathrm{Cl}^{-} \rightarrow \mathrm{HOCl}^{-} \\
& \mathrm{HOCl}^{-} \bullet+\mathrm{Cl}^{-} \rightarrow \mathrm{OH}^{-}+\mathrm{Cl}_{2}^{-} \bullet \\
& \mathrm{Cl} \bullet+\mathrm{Cl}^{-} \rightarrow \mathrm{Cl}_{2}^{-} \bullet \\
& \mathrm{HOBr}{ }^{\text {light }}>\mathrm{OH} \bullet+\mathrm{Br}^{\bullet} \\
& \mathrm{Br} \bullet+\mathrm{Br}^{-} \rightarrow \mathrm{Br}_{2}^{-} \\
& \mathrm{Cl} \bullet+\mathrm{Br}^{-} \rightarrow \mathrm{ClBr}^{-} \bullet
\end{aligned}
$$

The aqueous photodecomposition of $\mathrm{ClO}_{2}$ has been the subject of several studies (16-23) and more recently in nitrogen saturated aqueous solutions where they account quantitatively for the formation of $\mathrm{ClO}_{3}{ }^{-}$(24). Zika et al. (21) observed a rapid rate of $\mathrm{ClO}_{2}$ decomposition, with a 
reaction half life of less than 20 seconds in surface water in the absence of organic carbon, and proposed a reaction scheme that described the decomposition in aqueous solution:

$$
\begin{aligned}
& \mathrm{ClO}_{2}{ }^{\text {light }}>(\mathrm{ClO} \bullet+\mathrm{O} \bullet)_{\text {cage }} \\
& (\mathrm{ClO} \bullet+\mathrm{O} \bullet)_{\text {cage }} \rightarrow \mathrm{Cl} \bullet+\mathrm{O}_{2} \\
& \mathrm{ClO} \bullet+\mathrm{ClO}_{2} \rightarrow \mathrm{Cl}_{2} \mathrm{O}_{3} \\
& \mathrm{Cl}_{2} \mathrm{O}_{3}+\mathrm{H}_{2} \mathrm{O} \rightarrow \mathrm{ClO}^{-}+\mathrm{ClO}_{3^{-}}+2 \mathrm{H}^{+} \\
& \mathrm{Cl} \bullet+\mathrm{Cl}^{-} \rightarrow \mathrm{Cl}_{2} \bullet \\
& \mathrm{Cl} \bullet\left(\mathrm{or} \mathrm{Cl}_{2} \bullet \bullet+\mathrm{ClO}_{2} \rightarrow \mathrm{Cl}_{2} \mathrm{O}_{2}\right. \\
& \mathrm{Cl}_{2} \mathrm{O}_{2}+\mathrm{H}_{2} \mathrm{O} \rightarrow \mathrm{Cl}^{-}+\mathrm{ClO}_{3}{ }^{-}+2 \mathrm{H}^{+} \\
& \mathrm{O} \bullet+\mathrm{O}_{2} \rightarrow \mathrm{O}_{3}
\end{aligned}
$$

The effect of $\mathrm{Br}^{-}$on the decomposition of $\mathrm{ClO}_{2}$ was also reported by $\mathrm{Zika}$ et al. (21). In the absence of light $\mathrm{ClO}_{2}$ was stable and did not react with $\mathrm{Br}^{-}$, as no measurable change in absorbance was detected in $0.1 \mathrm{mM}$ solutions of $\mathrm{ClO}_{2}$. However, with greater than $1.3 \times 10^{-5} \mathrm{M}$ $\left(1 \mathrm{mg} \mathrm{L}^{-1}\right) \mathrm{Br}^{-}, \mathrm{ClO}_{2}$ decayed in the light and after the light was turned off, continued to decay in the dark, suggesting a photochemically initiated chain reaction.

Several of the intermediates in the photodecomposition of oxidants shown in equations 1 - 14 are known to react via insertion reactions with organic compounds (25). Photochlorination can occur in natural waters and result in the formation of halogenated by-products different than those in dark pathways (26-32), and similar reactions would presumably occur for bromine free radical species. Solutions of $\mathrm{ClO}_{2}$ in water containing $\mathrm{NOM}$ and bromide ion, when exposed to sunlight, showed a concentration dependent formation of bromoform (21). In addition, chlorite ion $\left(\mathrm{ClO}_{2}^{-}\right)$underwent photodecomposition, but at a much slower rate than $\mathrm{ClO}_{2}$, with $\mathrm{ClO}_{2}$ and $\mathrm{OCl}^{-}$formed as reaction products. 


\section{Methods and Materials}

Sunlight irradiation. Photochemical decomposition experiments were performed by exposing solutions of oxidant in Milli-Q water or GSW to sunlight in quartz tubes, submerged flush with the surface of distilled water $\left(25^{\circ} \mathrm{C}\right)$ to minimize heating. The loss of oxidant was monitored either directly by the decrease in absorbance of the oxidant or indirectly by iodometric titration (33). Oxidant concentration during sunlight experiments were recorded simultaneously with solar flux. Half lives and decomposition rates were calculated as first order decays. The solutions were studied at $\mathrm{pH}$ values ranging from $\mathrm{pH} 5-11$. The $\mathrm{pH}$ of the monochloramine solution was held constant at 8.5. The GSW had a pH of 8.1. The irradiations were conducted in Miami, FL, Lat. $24^{\circ} \mathrm{N}$ centered on solar noon when the solar flux was 0.40 watt-hr m${ }^{-2} \mathrm{hr}^{-1}$.

Results - Sunlight irradiation. The sunlight photolysis experiments showed that aqueous solutions of $\mathrm{HOCl} / \mathrm{OCl}^{-}$and $\mathrm{HOBr} / \mathrm{OBr}^{-}$undergo photodecomposition that is first order and the rate is $\mathrm{pH}$ dependent (Figure $\mathrm{S} 1$ ). 
A

$\mathrm{HOCl} / \mathrm{OCl}^{-}$

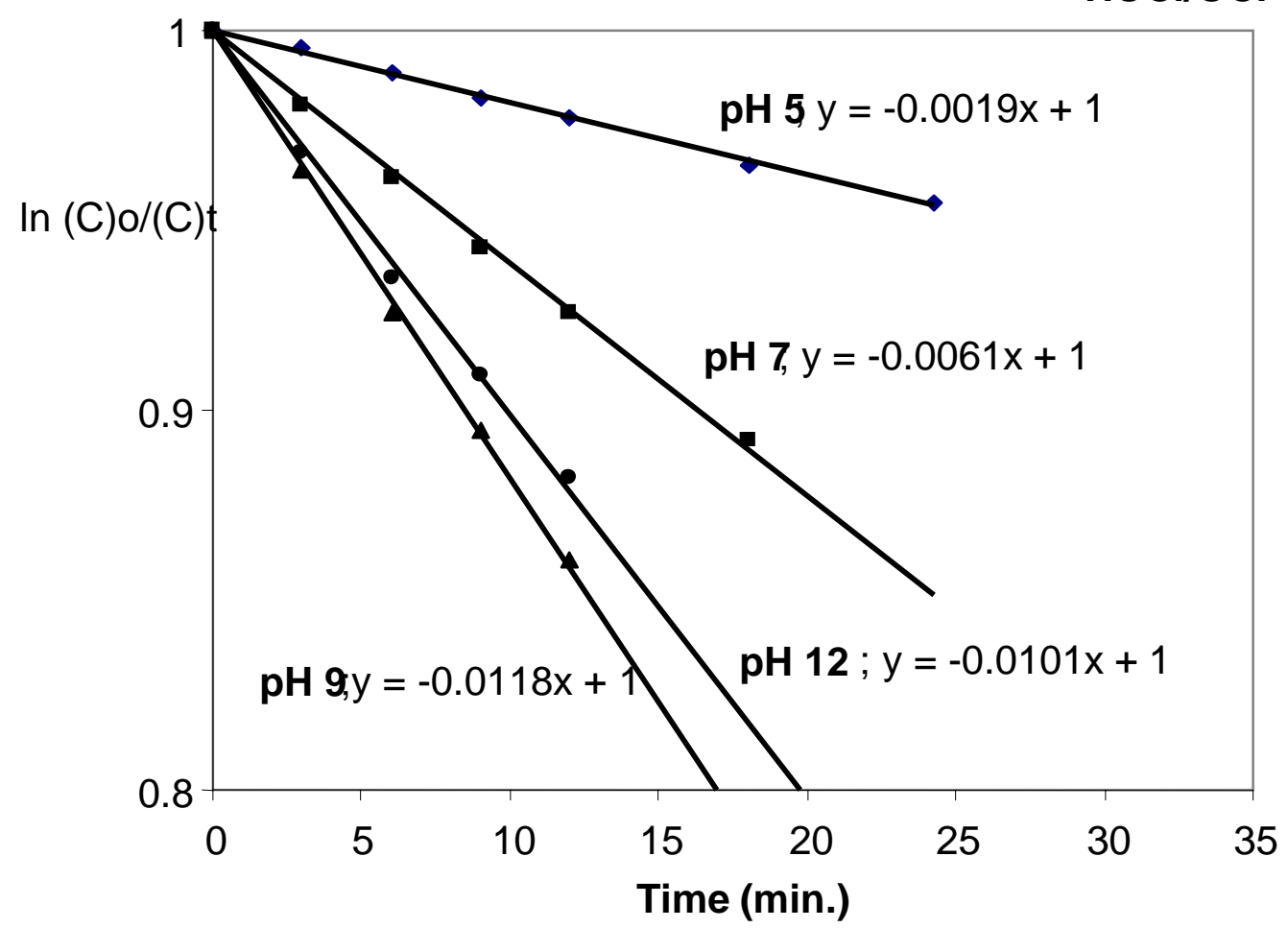

B

$\mathrm{HOBr} / \mathrm{OBr}^{-}$

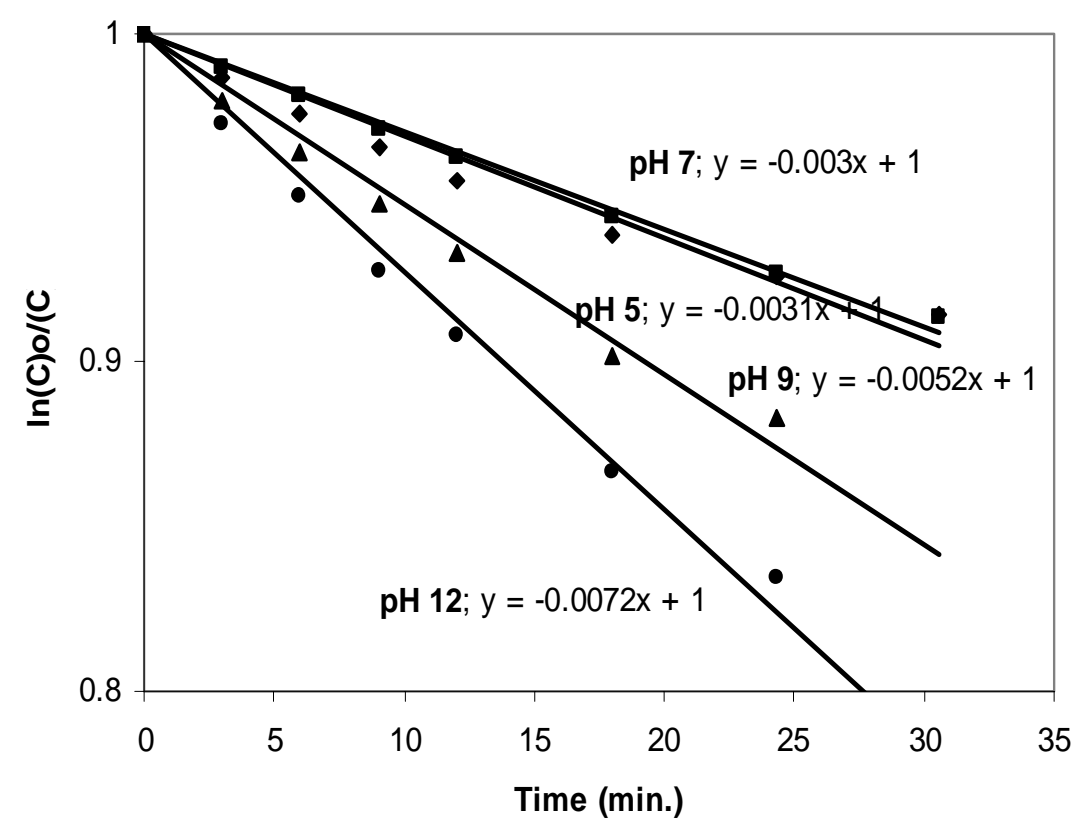

FIGURE S1. Sunlight decay of oxoacids and oxoanions of chlorine and bromine at various pHs in distilled water. (The irradiations were conducted in Miami, FL, Lat. $24{ }^{\circ} \mathrm{N}$ centered on solar noon when the solar flux was 0.40 watt-h $\mathrm{m}^{-2} \mathrm{~h}^{-1}$.) 


\section{References}

1. Buxton, G. V.; Subhani, M. S. Radiation chemistry and the photochemistry of oxychlorine ions. Part 1: Radiolysis of aqueous solutions of hypochlorite and chlorite ions. J. Chem. Soc. Faraday Trans. I. 1972, 68, 947-957.

2. Buxton, G. V.; Subhani, M. S. Radiation chemistry and the photochemistry of oxychlorine ions. Part 2: Photodecomposition of aqueous solutions of hypochlorite ions. J. Chem. Soc. Faraday Trans. I. 1972, 68, 958-969.

3. Buxton, G. V.; Subhani, M. S. Radiation chemistry and the photochemistry of oxychlorine ions. Part 3: Photodecomposition of aqueous solutions of chlorite ions. J. Chem. Soc. Faraday Trans. I. 1972, 68, 970-977.

4. Young, K. W.; Allmand, A. J. Experiments on the photolysis of aqueous solutions of chlorine, hypochlorous acid, and sodium hypochlorite. Canad. Jour. Res. 1949, 27B: $318-331$.

5. Kläning, U. K.; Wolff, T. Laser flash photolysis of $\mathrm{HClO}, \mathrm{ClO}^{-}, \mathrm{HBrO}$, and $\mathrm{BrO}^{-}$ in aqueous solution. Reactions of $\mathrm{Cl}^{-}$and $\mathrm{Br}^{-}$atoms. Ber. Bunsenges. Phys. Chem, 1985, $89,243-245$.

6. Hynes, A. J.; Wine, P. H. Time-resolved resonance Raman study of the spectroscopy and kinetics of the $\mathrm{Cl}_{2}{ }^{-}$radical anion in aqueous solution. J. Phys. Chem. 1988, 89, 35653572. 
7. Subhani, M. S.; Lodhi, S. F. A mechanism for the photolytic decomposition of hypobromite in aqueous solution at $365 \mathrm{~nm} 313 \mathrm{~nm}$ and $253.7 \mathrm{~nm}$. Revue Roumaine de Chemie, 1980, $25,1567-1578$.

8. Amichai, O.; Czapski, G.; Treinin, A. Flash photolysis of the oxybromine anions. Israel. Jour. Chem., 1969, 7, 351-359.

9. Amichai, O.; Treinin, A. Chemical reactivity of $\mathrm{O}\left({ }^{3} \mathrm{P}\right)$ atoms in aqueous solution. Chem. Phys. Letts., 1969, 3, 611-613.

10. Treinin, A. 1970. The photochemistry of oxyanions. Israel Jour. Chem., 1970, 8, 103-113.

11. Treinin, A.; Hayon, E. J. Charge transfer spectra of halogen atoms in water. Correlation of the electronic transition energies of iodine, bromine, chlorine, hydroxyl, and hydrogen radicals with their electron affinities. J. Am. Chem. Soc., 1975, 97, 1716-1721.

12. Kläning, U. K.; Sehested, K., Wolff, T. Ozone formation in laser flash photolysis of oxoacids and oxoanions of chlorine and bromine. J. Chem. Soc. Faraday Trans., 1984, 80, 2969-2979.

13. Macalady, D. L.; Carpenter, J. H.; Moore, C. A. Sunlight-induced bromate formation in chlorinated seawater. Science 1977, 195, 1335-1337.

14. Haag, W. R. The formation of N-bromo-N-chloroamines in chlorinated saline waters. Jour. Inorg. Nuc. Chem., 1980, 42, 1123-1127.

15. Wong, G. T. F. The effects of light on the dissipation of chlorine in sea-water. Water Res. 1980, $14,1263-1268$.

16. Bowen, E. J.; Cheung, W. M. The photodecomposition of chlorine dioxide solutions. J. Chem. Soc. 1932, 1200-1207.

17. Taube, H. Photochemical reactions of ozone in solution. Transactions of the 
Faraday Society, 1957, 53, 656-665.

18. Dardelet, S.; Robert, A. Stability of aqueous solutions of chlorine dioxide. Revue ATIP, 1968, 22, 123-128.

19. Mialocq, J. C.; Barat, F. Gilles, L.; Hickel, B.; Lesigne, B. Flash photolysis of chlorine dioxide in aqueous solution. J. Phys. Chem., 1973, 77, 742-749.

20. Shell-Sorokin, A. J.; Bethune, O. S.; Lankard, J. R.; Loy, M. M. T.; Sorokin, P. P. Chlorine perchlorate. A major photolysis product of chlorine dioxide. J. Phys. Chem., 1982, 86, 4653-4655.

21. Zika, R. G.; Moore, C. A.; Gidel, L. T.; Cooper, W. J. Sunlight-induced photodecomposition of chlorine dioxide. In, Jolley, R. L.; Bull, R. J.; Davis, W. P.; Katz, S.; Roberts, M. H., Jr.; Jacobs, V. A. (Eds.) Water Chlorination: Chemistry, Environmental Impact and Health Effects. Lewis Publishers., Inc., Chelsea, MI 1985, 5, 1041-1053.

22. Karpel vel Leitner, N.; De Laat, J.; Doré, M. Photodecomposition of chlorine dioxide and chlorite by U.V.-Irradiation-Part I. Photo-products. Water Res. 1992, 26, 1655-1664.

23. Karpel vel Leitner, N.; De Laat, J.; Doré, M. Photodecomposition of chlorine dioxide and chlorite by U.V.-Irradiation-Part I. Kinetic study. Water Res. 1992, 26, 1665-1672.

24. Quiroga, S. L.; Perissinotti, L. J. Reduced mechanism for the $366 \mathrm{~nm}$ chlorine dioxide photodecomposition in $\mathrm{N}_{2}$-saturated aqueous solutions. J. Photochem. Photobiol. A: Chem. 2005, 171, 59-67.

25. Hasegawa, K.; Neta, P. Rate constants and mechanisms of reaction of $\mathrm{Cl}_{2}{ }^{-}$radicals. J. Phys. Chem. 1978, 82, 854-857.

26. Jenkins, S. H. Recent investigations on the disappearance of chlorine in seawater. Mar. Pollut. Bull. 1981, 12, 398-399. 
27. Hancil, V.; Smith, J. M. Chlorine-sensitized photochemical oxidation of soluble organics in municipal waste water. Ind. Eng. Chem. Process. Des. Develop., 1971, 10, $515-523$.

28. Adams, G. E.; Aldrich, J. E.; Bisby, R. H.; Cundall, R. B.; Radpath, J. L.; Willson, R. L. elective free radical reactions with proteins and enzymes: Reactions of inorganic radical anions with amino acids. Radiat. Res., 1972, 49, 278-289.

29. Schorr, V; Boval, B.; Hancil, V.; Smith, J. M. Photooxidation kinetics of organic pollutants in municipal waste water. Ind. Eng. Chem. Process. Des. Develop., 1971, 10, 509-514.

30. Oliver, B. G.; Carey, J. H. Photochemical production of chlorinated organics in aqueous solutions containing chlorine. Environ. Sci. Tech., 1977, 11, 893-895.

31. Nakamura, M; Ogata, Y. Photolytic oxidation of alkylbenzenesulfonic acids by aqueous sodium hypochlorite. Bull. Chem. Soc. Japan., 1977, 50, 2396-2399.

32. Nowell, L. H.; Crosby, D. G. Photodegradation of water pollutants in chlorinated water. in Water Chlorination: Chemistry, Environmental Impact and Health Effects; Jolley, R. L.; Bull, R. J.; Davis, W. P.; Katz, S.; Roberts, M. H., Jr.; Jacobs, V. A. Eds.; Lewis Pub., Inc., Chelsea, MI, 1985, 5, 1055-1062.

33. Eaton, A. D.; Clesceri, L. S.; Greenberg, A. E. (Eds.) Standard Methods for the Examination of Water and Wastewater, $19^{\text {th }}$ Edition, APHA, AWWA, WEF, APHA, Washington DC, $1995,4-36-4-48$. 
TABLE S1. Photochemical (sunlight) half-life of $\mathrm{HOCl} \mathrm{OCl}-$ at latitude $40^{\circ}$, longitude $80^{\circ}$ in water with $44 \mu \mathrm{M}$ DOC as $\mathrm{C}\left(0.53 \mathrm{mg} \mathrm{L}^{-1}\right)$ in the winter and summer. Modeled data using GCSOLARS.

\begin{tabular}{|c|c|c|c|c|c|c|}
\hline $\begin{array}{l}\text { Depth } \\
\text { (m) }\end{array}$ & Zen. Ang. & $\begin{array}{c}\text { Time } \\
\text { (hours) }\end{array}$ & $\min$ & time & $\begin{array}{c}\mathrm{k} \\
\left(\mathrm{hr}^{-1}\right)\end{array}$ & $\begin{array}{l}\mathrm{T}_{1 / 2} \\
(\mathrm{hr})\end{array}$ \\
\hline \multicolumn{7}{|c|}{ Winter } \\
\hline \multirow[t]{9}{*}{0} & 0 & 7.54 & 32.4 & 732 & 0 & 0 \\
\hline & 5 & 8.12 & 7.2 & 807 & 0.0135 & 51.3 \\
\hline & 10 & 8.65 & 39 & 839 & 0.141 & 4.92 \\
\hline & 20 & 9.86 & 51.6 & 952 & 0.736 & 0.941 \\
\hline & 29.86 & 12.42 & 25.2 & 1225 & 1.58 & 0.438 \\
\hline & 20 & 14.92 & 55.2 & 1455 & 0.736 & 0.941 \\
\hline & 10 & 16.19 & 11.4 & 1611 & 0.141 & 4.92 \\
\hline & 5 & 16.71 & 42.6 & 1643 & 0.0135 & 51.3 \\
\hline & 0 & 17.29 & 17.4 & 1717 & 0 & 0 \\
\hline \multirow[t]{9}{*}{1} & 0 & 7.54 & 32.4 & 732 & 0 & 0 \\
\hline & 5 & 8.12 & 7.2 & 807 & 0.00555 & 125 \\
\hline & 10 & 8.65 & 39 & 839 & 0.054 & 12.8 \\
\hline & 20 & 9.86 & 51.6 & 952 & 0.26 & 2.66 \\
\hline & 29.86 & 12.42 & 25.2 & 1225 & 0.545 & 1.27 \\
\hline & 20 & 14.92 & 55.2 & 1455 & 0.26 & 2.66 \\
\hline & 10 & 16.19 & 11.4 & 1611 & 0.054 & 12.8 \\
\hline & 5 & 16.71 & 42.6 & 1643 & 0.00555 & 125 \\
\hline & 0 & 17.29 & 17.4 & 1717 & 0 & 0 \\
\hline \multirow[t]{9}{*}{2} & 0 & 7.54 & 32.4 & 732 & 0 & 0 \\
\hline & 5 & 8.12 & 7.2 & 807 & 0.00312 & 222 \\
\hline & 10 & 8.65 & 39 & 839 & 0.0298 & 23.2 \\
\hline & 20 & 9.86 & 51.6 & 952 & 0.141 & 4.92 \\
\hline & 29.86 & 12.42 & 25.2 & 1225 & 0.294 & 2.36 \\
\hline & 20 & 14.92 & 55.2 & 1455 & 0.141 & 4.92 \\
\hline & 10 & 16.19 & 11.4 & 1611 & 0.0298 & 23.2 \\
\hline & 5 & 16.71 & 42.6 & 1643 & 0.00312 & 222 \\
\hline & 0 & 17.29 & 17.4 & 1717 & 0 & 0 \\
\hline \multirow[t]{9}{*}{3} & 0 & 7.54 & 32.4 & 732 & 0 & 0 \\
\hline & 5 & 8.12 & 7.2 & 807 & 0.00211 & 329 \\
\hline & 10 & 8.65 & 39 & 839 & 0.0201 & 34.5 \\
\hline & 20 & 9.86 & 51.6 & 952 & 0.0947 & 7.32 \\
\hline & 29.86 & 12.42 & 25.2 & 1225 & 0.197 & 3.51 \\
\hline & 20 & 14.92 & 55.2 & 1455 & 0.0947 & 7.32 \\
\hline & 10 & 16.19 & 11.4 & 1611 & 0.0201 & 34.5 \\
\hline & 5 & 16.71 & 42.6 & 1643 & 0.00211 & 329 \\
\hline & 0 & 17.29 & 17.4 & 1717 & 0 & 0 \\
\hline \multirow[t]{2}{*}{4} & 0 & 7.54 & 32.4 & 732 & 0 & 0 \\
\hline & 5 & 8.12 & 7.2 & 807 & 0.00158 & 437 \\
\hline
\end{tabular}




\begin{tabular}{|c|c|c|c|c|c|c|}
\hline & 10 & 8.65 & 39 & 839 & 0.0151 & 46 \\
\hline & 20 & 9.86 & 51.6 & 952 & 0.0711 & 9.75 \\
\hline & 29.86 & 12.42 & 25.2 & 1225 & 0.148 & 4.68 \\
\hline & 20 & 14.92 & 55.2 & 1455 & 0.0711 & 9.75 \\
\hline & 10 & 16.19 & 11.4 & 1611 & 0.0151 & 46 \\
\hline & 5 & 16.71 & 42.6 & 1643 & 0.00158 & 437 \\
\hline & 0 & 17.29 & 17.4 & 1717 & 0 & 0 \\
\hline \multirow[t]{9}{*}{5} & 0 & 7.54 & 32.4 & 732 & 0 & 0 \\
\hline & 5 & 8.12 & 7.2 & 807 & 0.00127 & 546 \\
\hline & 10 & 8.65 & 39 & 839 & 0.0121 & 57.4 \\
\hline & 20 & 9.86 & 51.6 & 952 & 0.0569 & 12.2 \\
\hline & 29.86 & 12.42 & 25.2 & 1225 & 0.119 & 5.85 \\
\hline & 20 & 14.92 & 55.2 & 1455 & 0.0569 & 12.2 \\
\hline & 10 & 16.19 & 11.4 & 1611 & 0.0121 & 57.4 \\
\hline & 5 & 16.71 & 42.6 & 1643 & 0.00127 & 546 \\
\hline & 0 & 17.29 & 17.4 & 1717 & 0 & 0 \\
\hline $\begin{array}{l}\text { Depth } \\
(\mathrm{m})\end{array}$ & Zen. Ang. & $\begin{array}{c}\text { Time } \\
\text { (hours) }\end{array}$ & $\min$ & hours & $\begin{array}{c}\mathrm{k} \\
\left(\mathrm{hr}^{-1}\right)\end{array}$ & $\begin{array}{l}\mathrm{T}_{1 / 2} \\
\text { (hr) }\end{array}$ \\
\hline \multicolumn{7}{|c|}{ Summer } \\
\hline \multirow[t]{19}{*}{0} & 0 & 5.07 & 4.2 & 504 & 0 & 0 \\
\hline & 5 & 5.63 & 37.8 & 538 & 0.0136 & 51 \\
\hline & 10 & 6.09 & 5.4 & 605 & 0.142 & 4.89 \\
\hline & 20 & 6.99 & 59.4 & 659 & 0.741 & 0.936 \\
\hline & 30 & 7.86 & 51.6 & 752 & 1.6 & 0.432 \\
\hline & 40 & 8.73 & 43.8 & 844 & 2.5 & 0.277 \\
\hline & 50 & 9.62 & 37.2 & 937 & 3.3 & 0.21 \\
\hline & 60 & 10.58 & 34.8 & 1035 & 3.94 & 0.176 \\
\hline & 70 & 12.17 & 10.2 & 1210 & 4.4 & 0.158 \\
\hline & 70.09 & 12.32 & 19.2 & 1219 & 4.4 & 0.157 \\
\hline & 70 & 12.48 & 28.8 & 1229 & 4.4 & 0.158 \\
\hline & 60 & 14.07 & 4.2 & 1404 & 3.94 & 0.176 \\
\hline & 50 & 15.03 & 1.8 & 1502 & 3.3 & 0.21 \\
\hline & 40 & 15.92 & 55.2 & 1555 & 2.5 & 0.277 \\
\hline & 30 & 16.79 & 47.4 & 1647 & 1.6 & 0.432 \\
\hline & 20 & 17.66 & 39.6 & 1740 & 0.741 & 0.936 \\
\hline & 10 & 18.56 & 33.6 & 1834 & 0.142 & 4.89 \\
\hline & 5 & 19.02 & 1.2 & 1901 & 0.0136 & 51 \\
\hline & 0 & 19.58 & 34.8 & 1935 & 0 & 0 \\
\hline \multirow[t]{8}{*}{1} & 0 & 5.07 & 4.2 & 504 & 0 & 0 \\
\hline & 5 & 5.63 & 37.8 & 538 & 0.00558 & 124 \\
\hline & 10 & 6.09 & 5.4 & 605 & 0.0543 & 12.8 \\
\hline & 20 & 6.99 & 59.4 & 659 & 0.262 & 2.65 \\
\hline & 30 & 7.86 & 51.6 & 752 & 0.552 & 1.26 \\
\hline & 40 & 8.73 & 43.8 & 844 & 0.866 & 0.801 \\
\hline & 50 & 9.62 & 37.2 & 937 & 1.16 & 0.597 \\
\hline & 60 & 10.58 & 34.8 & 1035 & 1.41 & 0.492 \\
\hline
\end{tabular}




\begin{tabular}{|c|c|c|c|c|c|c|}
\hline & 70 & 12.17 & 10.2 & 1210 & 1.6 & 0.434 \\
\hline & 70.09 & 12.32 & 19.2 & 1219 & 1.6 & 0.434 \\
\hline & 70 & 12.48 & 28.8 & 1229 & 1.6 & 0.434 \\
\hline & 60 & 14.07 & 4.2 & 1404 & 1.41 & 0.492 \\
\hline & 50 & 15.03 & 1.8 & 1502 & 1.16 & 0.597 \\
\hline & 40 & 15.92 & 55.2 & 1555 & 0.866 & 0.801 \\
\hline & 30 & 16.79 & 47.4 & 1647 & 0.552 & 1.26 \\
\hline & 20 & 17.66 & 39.6 & 1740 & 0.262 & 2.65 \\
\hline & 10 & 18.56 & 33.6 & 1834 & 0.0543 & 12.8 \\
\hline & 5 & 19.02 & 1.2 & 1901 & 0.00558 & 124 \\
\hline & 0 & 19.58 & 34.8 & 1935 & 0 & 0 \\
\hline \multirow[t]{19}{*}{2} & 0 & 5.07 & 4.2 & 504 & 0 & 0 \\
\hline & 5 & 5.63 & 37.8 & 538 & 0.00313 & 221 \\
\hline & 10 & 6.09 & 5.4 & 605 & 0.0299 & 23.1 \\
\hline & 20 & 6.99 & 59.4 & 659 & 0.142 & 4.89 \\
\hline & 30 & 7.86 & 51.6 & 752 & 0.297 & 2.33 \\
\hline & 40 & 8.73 & 43.8 & 844 & 0.467 & 1.48 \\
\hline & 50 & 9.62 & 37.2 & 937 & 0.629 & 1.1 \\
\hline & 60 & 10.58 & 34.8 & 1035 & 0.768 & 0.902 \\
\hline & 70 & 12.17 & 10.2 & 1210 & 0.874 & 0.793 \\
\hline & 70.09 & 12.32 & 19.2 & 1219 & 0.874 & 0.792 \\
\hline & 70 & 12.48 & 28.8 & 1229 & 0.874 & 0.793 \\
\hline & 60 & 14.07 & 4.2 & 1404 & 0.768 & 0.902 \\
\hline & 50 & 15.03 & 1.8 & 1502 & 0.629 & 1.1 \\
\hline & 40 & 15.92 & 55.2 & 1555 & 0.467 & 1.48 \\
\hline & 30 & 16.79 & 47.4 & 1647 & 0.297 & 2.33 \\
\hline & 20 & 17.66 & 39.6 & 1740 & 0.142 & 4.89 \\
\hline & 10 & 18.56 & 33.6 & 1834 & 0.0299 & 23.1 \\
\hline & 5 & 19.02 & 1.2 & 1901 & 0.00313 & 221 \\
\hline & 0 & 19.58 & 34.8 & 1935 & 0 & 0 \\
\hline \multirow[t]{16}{*}{3} & 0 & 5.07 & 4.2 & 504 & 0 & 0 \\
\hline & 5 & 5.63 & 37.8 & 538 & 0.00212 & 327 \\
\hline & 10 & 6.09 & 5.4 & 605 & 0.0202 & 34.3 \\
\hline & 20 & 6.99 & 59.4 & 659 & 0.0952 & 7.28 \\
\hline & 30 & 7.86 & 51.6 & 752 & 0.2 & 3.47 \\
\hline & 40 & 8.73 & 43.8 & 844 & 0.314 & 2.21 \\
\hline & 50 & 9.62 & 37.2 & 937 & 0.423 & 1.64 \\
\hline & 60 & 10.58 & 34.8 & 1035 & 0.517 & 1.34 \\
\hline & 70 & 12.17 & 10.2 & 1210 & 0.589 & 1.18 \\
\hline & 70.09 & 12.32 & 19.2 & 1219 & 0.589 & 1.18 \\
\hline & 70 & 12.48 & 28.8 & 1229 & 0.589 & 1.18 \\
\hline & 60 & 14.07 & 4.2 & 1404 & 0.517 & 1.34 \\
\hline & 50 & 15.03 & 1.8 & 1502 & 0.423 & 1.64 \\
\hline & 40 & 15.92 & 55.2 & 1555 & 0.314 & 2.21 \\
\hline & 30 & 16.79 & 47.4 & 1647 & 0.2 & 3.47 \\
\hline & 20 & 17.66 & 39.6 & 1740 & 0.0952 & 7.28 \\
\hline
\end{tabular}




\begin{tabular}{|c|c|c|c|c|c|c|}
\hline & 10 & 18.56 & 33.6 & 1834 & 0.0202 & 34.3 \\
\hline & 5 & 19.02 & 1.2 & 1901 & 0.00212 & 327 \\
\hline & 0 & 19.58 & 34.8 & 1935 & 0 & 0 \\
\hline \multirow[t]{19}{*}{4} & 0 & 5.07 & 4.2 & 504 & 0 & 0 \\
\hline & 5 & 5.63 & 37.8 & 538 & 0.00159 & 435 \\
\hline & 10 & 6.09 & 5.4 & 605 & 0.0152 & 45.7 \\
\hline & 20 & 6.99 & 59.4 & 659 & 0.0714 & 9.7 \\
\hline & 30 & 7.86 & 51.6 & 752 & 0.15 & 4.62 \\
\hline & 40 & 8.73 & 43.8 & 844 & 0.236 & 2.94 \\
\hline & 50 & 9.62 & 37.2 & 937 & 0.318 & 2.18 \\
\hline & 60 & 10.58 & 34.8 & 1035 & 0.388 & 1.78 \\
\hline & 70 & 12.17 & 10.2 & 1210 & 0.442 & 1.57 \\
\hline & 70.09 & 12.32 & 19.2 & 1219 & 0.443 & 1.57 \\
\hline & 70 & 12.48 & 28.8 & 1229 & 0.442 & 1.57 \\
\hline & 60 & 14.07 & 4.2 & 1404 & 0.388 & 1.78 \\
\hline & 50 & 15.03 & 1.8 & 1502 & 0.318 & 2.18 \\
\hline & 40 & 15.92 & 55.2 & 1555 & 0.236 & 2.94 \\
\hline & 30 & 16.79 & 47.4 & 1647 & 0.15 & 4.62 \\
\hline & 20 & 17.66 & 39.6 & 1740 & 0.0714 & 9.7 \\
\hline & 10 & 18.56 & 33.6 & 1834 & 0.0152 & 45.7 \\
\hline & 5 & 19.02 & 1.2 & 1901 & 0.00159 & 435 \\
\hline & 0 & 19.58 & 34.8 & 1935 & 0 & 0 \\
\hline \multirow[t]{19}{*}{5} & 0 & 5.07 & 4.2 & 504 & 0 & 0 \\
\hline & 5 & 5.63 & 37.8 & 538 & 0.00127 & 544 \\
\hline & 10 & 6.09 & 5.4 & 605 & 0.0121 & 57.2 \\
\hline & 20 & 6.99 & 59.4 & 659 & 0.0572 & 12.1 \\
\hline & 30 & 7.86 & 51.6 & 752 & 0.12 & 5.78 \\
\hline & 40 & 8.73 & 43.8 & 844 & 0.188 & 3.68 \\
\hline & 50 & 9.62 & 37.2 & 937 & 0.254 & 2.73 \\
\hline & 60 & 10.58 & 34.8 & 1035 & 0.311 & 2.23 \\
\hline & 70 & 12.17 & 10.2 & 1210 & 0.354 & 1.96 \\
\hline & 70.09 & 12.32 & 19.2 & 1219 & 0.354 & 1.96 \\
\hline & 70 & 12.48 & 28.8 & 1229 & 0.354 & 1.96 \\
\hline & 60 & 14.07 & 4.2 & 1404 & 0.311 & 2.23 \\
\hline & 50 & 15.03 & 1.8 & 1502 & 0.254 & 2.73 \\
\hline & 40 & 15.92 & 55.2 & 1555 & 0.188 & 3.68 \\
\hline & 30 & 16.79 & 47.4 & 1647 & 0.12 & 5.78 \\
\hline & 20 & 17.66 & 39.6 & 1740 & 0.0572 & 12.1 \\
\hline & 10 & 18.56 & 33.6 & 1834 & 0.0121 & 57.2 \\
\hline & 5 & 19.02 & 1.2 & 1901 & 0.00127 & 544 \\
\hline & 0 & 19.58 & 34.8 & 1935 & 0 & 0 \\
\hline
\end{tabular}


TABLE S2. Photochemical (sunlight) half-life of $\mathrm{HOCl} / \mathrm{OCl}^{-}$at latitude $40^{\circ}$, longitude $80^{\circ}$ in water with $1.47 \mathrm{mM} \mathrm{DOC}$ as $\mathrm{C}\left(17.6 \mathrm{mg} \mathrm{L}^{-1}\right)$ in the winter and summer. Modeled data using GCSOLARS.

\begin{tabular}{|c|c|c|c|c|c|c|}
\hline $\begin{array}{l}\text { Depth } \\
(\mathrm{m})\end{array}$ & Zen. Ang. & $\begin{array}{l}\text { Time } \\
\text { (hours) }\end{array}$ & $\min$ & hours & $\begin{array}{c}\mathrm{k} \\
\left(\mathrm{hr}^{-1}\right)\end{array}$ & $\begin{array}{l}\mathrm{T}_{1 / 2} \\
(\mathrm{hr})\end{array}$ \\
\hline \multicolumn{7}{|c|}{ Winter } \\
\hline \multirow[t]{9}{*}{0} & 0 & 7.54 & 32.4 & 732 & 0 & 0 \\
\hline & 5 & 8.12 & 7.2 & 807 & 0.0135 & 51.3 \\
\hline & 10 & 8.65 & 39 & 839 & 0.141 & 4.92 \\
\hline & 20 & 9.86 & 51.6 & 952 & 0.736 & 0.942 \\
\hline & 29.86 & 12.42 & 25.2 & 1225 & 1.58 & 0.438 \\
\hline & 20 & 14.92 & 55.2 & 1455 & 0.736 & 0.942 \\
\hline & 10 & 16.19 & 11.4 & 1611 & 0.141 & 4.92 \\
\hline & 5 & 16.71 & 42.6 & 1643 & 0.0135 & 51.3 \\
\hline & 0 & 17.29 & 17.4 & 1717 & 0 & \\
\hline \multirow[t]{9}{*}{1} & 0 & 7.54 & 32.4 & 732 & 0 & 0 \\
\hline & 5 & 8.12 & 7.2 & 807 & 0.000237 & 2,920 \\
\hline & 10 & 8.65 & 39 & 839 & 0.00224 & 310 \\
\hline & 20 & 9.86 & 51.6 & 952 & 0.0106 & 65.5 \\
\hline & 29.86 & 12.42 & 25.2 & 1225 & 0.0222 & 31.3 \\
\hline & 20 & 14.92 & 55.2 & 1455 & 0.0106 & 65.5 \\
\hline & 10 & 16.19 & 11.4 & 1611 & 0.00224 & 310 \\
\hline & 5 & 16.71 & 42.6 & 1643 & 0.000237 & 2,920 \\
\hline & 0 & 17.29 & 17.4 & 1717 & 0 & \\
\hline \multirow[t]{9}{*}{2} & 0 & 7.54 & 32.4 & 732 & 0 & 0 \\
\hline & 5 & 8.12 & 7.2 & 807 & 0.000119 & 5,840 \\
\hline & 10 & 8.65 & 39 & 839 & 0.00112 & 620 \\
\hline & 20 & 9.86 & 51.6 & 952 & 0.00529 & 131 \\
\hline & 29.86 & 12.42 & 25.2 & 1225 & 0.0111 & 62.5 \\
\hline & 20 & 14.92 & 55.2 & 1455 & 0.00529 & 131 \\
\hline & 10 & 16.19 & 11.4 & 1611 & 0.00112 & 620 \\
\hline & 5 & 16.71 & 42.6 & 1643 & 0.000119 & 5,840 \\
\hline & 0 & 17.29 & 17.4 & 1717 & 0 & \\
\hline \multirow[t]{9}{*}{3} & 0 & 7.54 & 32.4 & 732 & 0 & 0 \\
\hline & 5 & 8.12 & 7.2 & 807 & 0.0000791 & 8,770 \\
\hline & 10 & 8.65 & 39 & 839 & 0.000746 & 930 \\
\hline & 20 & 9.86 & 51.6 & 952 & 0.00353 & 196 \\
\hline & 29.86 & 12.42 & 25.2 & 1225 & 0.00739 & 93.8 \\
\hline & 20 & 14.92 & 55.2 & 1455 & 0.00353 & 196 \\
\hline & 10 & 16.19 & 11.4 & 1611 & 0.000746 & 930 \\
\hline & 5 & 16.71 & 42.6 & 1643 & 0.0000791 & 8,770 \\
\hline & 0 & 17.29 & 17.4 & 1717 & 0 & \\
\hline & & & & & & \\
\hline
\end{tabular}




\begin{tabular}{|c|c|c|c|c|c|c|}
\hline $\begin{array}{l}\text { Depth } \\
\text { (m) }\end{array}$ & Zen. Ang. & $\begin{array}{c}\text { Time } \\
\text { (hours) }\end{array}$ & $\min$ & hours & $\begin{array}{c}\mathrm{k} \\
(\mathrm{hr}-1)\end{array}$ & $\begin{array}{l}\mathrm{T}_{1 / 2} \\
(\mathrm{hr})\end{array}$ \\
\hline \multicolumn{7}{|c|}{ Summer } \\
\hline \multirow[t]{19}{*}{0} & 0 & 5.07 & 4.2 & 504 & 0 & 0 \\
\hline & 5 & 5.63 & 37.8 & 538 & 0.0136 & 51.1 \\
\hline & 10 & 6.09 & 5.4 & 605 & 0.142 & 4.9 \\
\hline & 20 & 6.99 & 59.4 & 659 & 0.74 & 0.936 \\
\hline & 30 & 7.86 & 51.6 & 752 & 1.6 & 0.432 \\
\hline & 40 & 8.73 & 43.8 & 844 & 2.5 & 0.277 \\
\hline & 50 & 9.62 & 37.2 & 937 & 3.3 & 0.21 \\
\hline & 60 & 10.58 & 34.8 & 1035 & 3.94 & 0.176 \\
\hline & 70 & 12.17 & 10.2 & 1210 & 4.4 & 0.158 \\
\hline & 70.09 & 12.32 & 19.2 & 1219 & 4.4 & 0.157 \\
\hline & 70 & 12.48 & 28.8 & 1229 & 4.4 & 0.158 \\
\hline & 60 & 14.07 & 4.2 & 1404 & 3.94 & 0.176 \\
\hline & 50 & 15.03 & 1.8 & 1502 & 3.3 & 0.21 \\
\hline & 40 & 15.92 & 55.2 & 1555 & 2.5 & 0.277 \\
\hline & 30 & 16.79 & 47.4 & 1647 & 1.6 & 0.432 \\
\hline & 20 & 17.66 & 39.6 & 1740 & 0.74 & 0.936 \\
\hline & 10 & 18.56 & 33.6 & 1834 & 0.142 & 4.9 \\
\hline & 5 & 19.02 & 1.2 & 1901 & 0.0136 & 51.1 \\
\hline & 0 & 19.58 & 34.8 & 1935 & 0 & \\
\hline \multirow[t]{19}{*}{1} & 0 & 5.07 & 4.2 & 504 & 0 & 0 \\
\hline & 5 & 5.63 & 37.8 & 538 & 0.000238 & 2,910 \\
\hline & 10 & 6.09 & 5.4 & 605 & 0.00225 & 308 \\
\hline & 20 & 6.99 & 59.4 & 659 & 0.0106 & 65.1 \\
\hline & 30 & 7.86 & 51.6 & 752 & 0.0224 & 30.9 \\
\hline & 40 & 8.73 & 43.8 & 844 & 0.0354 & 19.6 \\
\hline & 50 & 9.62 & 37.2 & 937 & 0.0479 & 14.5 \\
\hline & 60 & 10.58 & 34.8 & 1035 & 0.0587 & 11.8 \\
\hline & 70 & 12.17 & 10.2 & 1210 & 0.067 & 10.3 \\
\hline & 70.09 & 12.32 & 19.2 & 1219 & 0.067 & 10.3 \\
\hline & 70 & 12.48 & 28.8 & 1229 & 0.067 & 10.3 \\
\hline & 60 & 14.07 & 4.2 & 1404 & 0.0587 & 11.8 \\
\hline & 50 & 15.03 & 1.8 & 1502 & 0.0479 & 14.5 \\
\hline & 40 & 15.92 & 55.2 & 1555 & 0.0354 & 19.6 \\
\hline & 30 & 16.79 & 47.4 & 1647 & 0.0224 & 30.9 \\
\hline & 20 & 17.66 & 39.6 & 1740 & 0.0106 & 65.1 \\
\hline & 10 & 18.56 & 33.6 & 1834 & 0.00225 & 308 \\
\hline & 5 & 19.02 & 1.2 & 1901 & 0.000238 & 2,910 \\
\hline & 0 & 19.58 & 34.8 & 1935 & 0 & \\
\hline \multirow[t]{5}{*}{2} & 0 & 5.07 & 4.2 & 504 & 0 & 0 \\
\hline & 5 & 5.63 & 37.8 & 538 & 0.000119 & 5,820 \\
\hline & 10 & 6.09 & 5.4 & 605 & 0.00112 & 617 \\
\hline & 20 & 6.99 & 59.4 & 659 & 0.00532 & 130 \\
\hline & 30 & 7.86 & 51.6 & 752 & 0.0112 & 61.8 \\
\hline
\end{tabular}




\begin{tabular}{|c|c|c|c|c|c|c|}
\hline & 40 & 8.73 & 43.8 & 844 & 0.0177 & 39.1 \\
\hline & 50 & 9.62 & 37.2 & 937 & 0.0239 & 28.9 \\
\hline & 60 & 10.58 & 34.8 & 1035 & 0.0293 & 23.6 \\
\hline & 70 & 12.17 & 10.2 & 1210 & 0.0335 & 20.7 \\
\hline & 70.09 & 12.32 & 19.2 & 1219 & 0.0335 & 20.7 \\
\hline & 70 & 12.48 & 28.8 & 1229 & 0.0335 & 20.7 \\
\hline & 60 & 14.07 & 4.2 & 1404 & 0.0293 & 23.6 \\
\hline & 50 & 15.03 & 1.8 & 1502 & 0.0239 & 28.9 \\
\hline & 40 & 15.92 & 55.2 & 1555 & 0.0177 & 39.1 \\
\hline & 30 & 16.79 & 47.4 & 1647 & 0.0112 & 61.8 \\
\hline & 20 & 17.66 & 39.6 & 1740 & 0.00532 & 130 \\
\hline & 10 & 18.56 & 33.6 & 1834 & 0.00112 & 617 \\
\hline & 5 & 19.02 & 1.2 & 1901 & 0.000119 & 5,820 \\
\hline & 0 & 19.58 & 34.8 & 1935 & 0 & \\
\hline \multirow[t]{19}{*}{3} & 0 & 5.07 & 4.2 & 504 & 0 & 0 \\
\hline & 5 & 5.63 & 37.8 & 538 & 0.0000794 & 8,730 \\
\hline & 10 & 6.09 & 5.4 & 605 & 0.000749 & 925 \\
\hline & 20 & 6.99 & 59.4 & 659 & 0.00355 & 195 \\
\hline & 30 & 7.86 & 51.6 & 752 & 0.00748 & 92.6 \\
\hline & 40 & 8.73 & 43.8 & 844 & 0.0118 & 58.7 \\
\hline & 50 & 9.62 & 37.2 & 937 & 0.016 & 43.4 \\
\hline & 60 & 10.58 & 34.8 & 1035 & 0.0196 & 35.4 \\
\hline & 70 & 12.17 & 10.2 & 1210 & 0.0223 & 31 \\
\hline & 70.09 & 12.32 & 19.2 & 1219 & 0.0223 & 31 \\
\hline & 70 & 12.48 & 28.8 & 1229 & 0.0223 & 31 \\
\hline & 60 & 14.07 & 4.2 & 1404 & 0.0196 & 35.4 \\
\hline & 50 & 15.03 & 1.8 & 1502 & 0.016 & 43.4 \\
\hline & 40 & 15.92 & 55.2 & 1555 & 0.0118 & 58.7 \\
\hline & 30 & 16.79 & 47.4 & 1647 & 0.00748 & 92.6 \\
\hline & 20 & 17.66 & 39.6 & 1740 & 0.00355 & 195 \\
\hline & 10 & 18.56 & 33.6 & 1834 & 0.000749 & 925 \\
\hline & 5 & 19.02 & 1.2 & 1901 & 0.0000794 & 8,730 \\
\hline & 0 & 19.58 & 34.8 & 1935 & 0 & \\
\hline
\end{tabular}


TABLE S3. Photochemical (sunlight) half-life of $\mathrm{HOBr} / \mathrm{OBr}^{-}$at latitude $40^{\circ}$, longitude $80^{\circ}$ in water with $1.47 \mathrm{mM} \mathrm{DOC}$ as $\mathrm{C}\left(17.6 \mathrm{mg} \mathrm{L}^{-1}\right)$ in the winter and summer. Modeled data using GCSOLARS.

\begin{tabular}{|c|c|c|c|c|c|c|}
\hline $\begin{array}{l}\text { Depth } \\
(\mathrm{m})\end{array}$ & Zen. Ang. & $\begin{array}{c}\text { Time } \\
\text { (hours) }\end{array}$ & Min & hours & $\begin{array}{c}\mathrm{k} \\
\left(\mathrm{hr}^{-1}\right)\end{array}$ & $\begin{array}{l}T_{1 / 2} \\
(h r)\end{array}$ \\
\hline \multicolumn{7}{|c|}{ Winter } \\
\hline \multirow[t]{9}{*}{0} & 0 & 7.54 & 32.4 & 732 & 0 & 0 \\
\hline & 5 & 8.12 & 7.2 & 807 & 0.0251 & 32.3 \\
\hline & 10 & 8.65 & 39 & 839 & 0.128 & 5.4 \\
\hline & 20 & 9.86 & 51.6 & 952 & 0.496 & 1.4 \\
\hline & 29.86 & 12.42 & 25.2 & 1225 & 0.929 & 0.746 \\
\hline & 20 & 14.92 & 55.2 & 1455 & 0.496 & 1.4 \\
\hline & 10 & 16.19 & 11.4 & 1611 & 0.128 & 5.4 \\
\hline & 5 & 16.71 & 42.6 & 1643 & 0.0251 & 32.3 \\
\hline & 0 & 17.29 & 17.4 & 1717 & 0 & \\
\hline \multirow[t]{9}{*}{1} & 0 & 7.54 & 32.4 & 732 & 0 & 0 \\
\hline & 5 & 8.12 & 7.2 & 807 & 0.000617 & 1,120 \\
\hline & 10 & 8.65 & 39 & 839 & 0.00288 & 241 \\
\hline & 20 & 9.86 & 51.6 & 952 & 0.00974 & 71.1 \\
\hline & 29.86 & 12.42 & 25.2 & 1225 & 0.0179 & 38.8 \\
\hline & 20 & 14.92 & 55.2 & 1455 & 0.00974 & 71.1 \\
\hline & 10 & 16.19 & 11.4 & 1611 & 0.00288 & 241 \\
\hline & 5 & 16.71 & 42.6 & 1643 & 0.000617 & 1,120 \\
\hline & 0 & 17.29 & 17.4 & 1717 & 0 & \\
\hline \multirow[t]{9}{*}{2} & 0 & 7.54 & 32.4 & 732 & 0 & 0 \\
\hline & 5 & 8.12 & 7.2 & 807 & 0.000308 & 2,250 \\
\hline & 10 & 8.65 & 39 & 839 & 0.00144 & 482 \\
\hline & 20 & 9.86 & 51.6 & 952 & 0.00487 & 142 \\
\hline & 29.86 & 12.42 & 25.2 & 1225 & 0.00893 & 77.6 \\
\hline & 20 & 14.92 & 55.2 & 1455 & 0.00487 & 142 \\
\hline & 10 & 16.19 & 11.4 & 1611 & 0.00144 & 482 \\
\hline & 5 & 16.71 & 42.6 & 1643 & 0.000308 & 2,250 \\
\hline & 0 & 17.29 & 17.4 & 1717 & 0 & \\
\hline \multirow[t]{9}{*}{3} & 0 & 7.54 & 32.4 & 732 & 0 & 0 \\
\hline & 5 & 8.12 & 7.2 & 807 & 0.000206 & 3,370 \\
\hline & 10 & 8.65 & 39 & 839 & 0.000959 & 722 \\
\hline & 20 & 9.86 & 51.6 & 952 & 0.00325 & 213 \\
\hline & 29.86 & 12.42 & 25.2 & 1225 & 0.00595 & 116 \\
\hline & 20 & 14.92 & 55.2 & 1455 & 0.00325 & 213 \\
\hline & 10 & 16.19 & 11.4 & 1611 & 0.000959 & 722 \\
\hline & 5 & 16.71 & 42.6 & 1643 & 0.000206 & 3,370 \\
\hline & 0 & 17.29 & 17.4 & 1717 & 0 & \\
\hline \multirow[t]{2}{*}{4} & 0 & 7.54 & 32.4 & 732 & 0 & 0 \\
\hline & 5 & 8.12 & 7.2 & 807 & 0.000154 & 4,490 \\
\hline
\end{tabular}




\begin{tabular}{|c|c|c|c|c|c|c|}
\hline & 10 & 8.65 & 39 & 839 & 0.000719 & 963 \\
\hline & 20 & 9.86 & 51.6 & 952 & 0.00244 & 284 \\
\hline & 29.86 & 12.42 & 25.2 & 1225 & 0.00446 & 155 \\
\hline & 20 & 14.92 & 55.2 & 1455 & 0.00244 & 284 \\
\hline & 10 & 16.19 & 11.4 & 1611 & 0.000719 & 963 \\
\hline & 5 & 16.71 & 42.6 & 1643 & 0.000154 & 4,490 \\
\hline & 0 & 17.29 & 17.4 & 1717 & 0 & \\
\hline \multirow[t]{9}{*}{5} & 0 & 7.54 & 32.4 & 732 & 0 & 0 \\
\hline & 5 & 8.12 & 7.2 & 807 & 0.000123 & 5,620 \\
\hline & 10 & 8.65 & 39 & 839 & 0.000576 & 1,200 \\
\hline & 20 & 9.86 & 51.6 & 952 & 0.00195 & 356 \\
\hline & 29.86 & 12.42 & 25.2 & 1225 & 0.00357 & 194 \\
\hline & 20 & 14.92 & 55.2 & 1455 & 0.00195 & 356 \\
\hline & 10 & 16.19 & 11.4 & 1611 & 0.000576 & 1,200 \\
\hline & 5 & 16.71 & 42.6 & 1643 & 0.000123 & 5,620 \\
\hline & 0 & 17.29 & 17.4 & 1717 & 0 & \\
\hline $\begin{array}{c}\text { Depth } \\
(\mathrm{m})\end{array}$ & Zen. Ang. & $\begin{array}{c}\text { Time } \\
\text { (hours) }\end{array}$ & $\min$ & hours & $\begin{array}{c}\mathrm{k} \\
\left(\mathrm{hr}^{-1}\right)\end{array}$ & $\begin{array}{l}\mathrm{T}_{1 / 2} \\
(\mathrm{hr})\end{array}$ \\
\hline \multicolumn{7}{|c|}{ Summer } \\
\hline \multirow[t]{19}{*}{0} & 0 & 5.07 & 4.2 & 504 & 0 & 0 \\
\hline & 5 & 5.63 & 37.8 & 538 & 0.0215 & 32.2 \\
\hline & 10 & 6.09 & 5.4 & 605 & 0.129 & 5.38 \\
\hline & 20 & 6.99 & 59.4 & 659 & 0.497 & 1.39 \\
\hline & 30 & 7.86 & 51.6 & 752 & 0.937 & 0.74 \\
\hline & 40 & 8.73 & 43.8 & 844 & 1.34 & 0.516 \\
\hline & 50 & 9.62 & 37.2 & 937 & 1.67 & 0.415 \\
\hline & 60 & 10.58 & 34.8 & 1035 & 1.92 & 0.362 \\
\hline & 70 & 12.17 & 10.2 & 1210 & 2.08 & 0.332 \\
\hline & 70.09 & 12.32 & 19.2 & 1219 & 2.09 & 0.332 \\
\hline & 70 & 12.48 & 28.8 & 1229 & 2.08 & 0.332 \\
\hline & 60 & 14.07 & 4.2 & 1404 & 1.92 & 0.362 \\
\hline & 50 & 15.03 & 1.8 & 1502 & 1.67 & 0.415 \\
\hline & 40 & 15.92 & 55.2 & 1555 & 1.34 & 0.516 \\
\hline & 30 & 16.79 & 47.4 & 1647 & 0.937 & 0.74 \\
\hline & 20 & 17.66 & 39.6 & 1740 & 0.497 & 1.39 \\
\hline & 10 & 18.56 & 33.6 & 1834 & 0.129 & 5.38 \\
\hline & 5 & 19.02 & 1.2 & 1901 & 0.0215 & 32.2 \\
\hline & 0 & 19.58 & 34.8 & 1935 & 0 & 0 \\
\hline \multirow[t]{8}{*}{1} & 0 & 5.07 & 4.2 & 504 & 0 & 0 \\
\hline & 5 & 5.63 & 37.8 & 538 & 0.000617 & 1,120 \\
\hline & 10 & 6.09 & 5.4 & 605 & 0.00288 & 240 \\
\hline & 20 & 6.99 & 59.4 & 659 & 0.00976 & 71 \\
\hline & 30 & 7.86 & 51.6 & 752 & 0.018 & 38.5 \\
\hline & 40 & 8.73 & 43.8 & 844 & 0.0262 & 26.4 \\
\hline & 50 & 9.62 & 37.2 & 937 & 0.0336 & 20.6 \\
\hline & 60 & 10.58 & 34.8 & 1035 & 0.0398 & 17.4 \\
\hline
\end{tabular}




\begin{tabular}{|c|c|c|c|c|c|c|}
\hline & 70 & 12.17 & 10.2 & 1210 & 0.0444 & 15.6 \\
\hline & 70.09 & 12.32 & 19.2 & 1219 & 0.0444 & 15.6 \\
\hline & 70 & 12.48 & 28.8 & 1229 & 0.0444 & 15.6 \\
\hline & 60 & 14.07 & 4.2 & 1404 & 0.0398 & 17.4 \\
\hline & 50 & 15.03 & 1.8 & 1502 & 0.0336 & 20.6 \\
\hline & 40 & 15.92 & 55.2 & 1555 & 0.0262 & 26.4 \\
\hline & 30 & 16.79 & 47.4 & 1647 & 0.018 & 38.5 \\
\hline & 20 & 17.66 & 39.6 & 1740 & 0.00976 & 71 \\
\hline & 10 & 18.56 & 33.6 & 1834 & 0.00288 & 240 \\
\hline & 5 & 19.02 & 1.2 & 1901 & 0.000617 & 1,120 \\
\hline & 0 & 19.58 & 34.8 & 1935 & 0 & 0 \\
\hline \multirow[t]{19}{*}{2} & 0 & 5.07 & 4.2 & 504 & 0 & 0 \\
\hline & 5 & 5.63 & 37.8 & 538 & 0.000309 & 2,240 \\
\hline & 10 & 6.09 & 5.4 & 605 & 0.00144 & 481 \\
\hline & 20 & 6.99 & 59.4 & 659 & 0.00488 & 142 \\
\hline & 30 & 7.86 & 51.6 & 752 & 0.00899 & 77 \\
\hline & 40 & 8.73 & 43.8 & 844 & 0.0131 & 52.9 \\
\hline & 50 & 9.62 & 37.2 & 937 & 0.0168 & 41.2 \\
\hline & 60 & 10.58 & 34.8 & 1035 & 0.0199 & 34.8 \\
\hline & 70 & 12.17 & 10.2 & 1210 & 0.0222 & 31.2 \\
\hline & 70.09 & 12.32 & 19.2 & 1219 & 0.0222 & 31.2 \\
\hline & 70 & 12.48 & 28.8 & 1229 & 0.0222 & 31.2 \\
\hline & 60 & 14.07 & 4.2 & 1404 & 0.0199 & 34.8 \\
\hline & 50 & 15.03 & 1.8 & 1502 & 0.0168 & 41.2 \\
\hline & 40 & 15.92 & 55.2 & 1555 & 0.0131 & 52.9 \\
\hline & 30 & 16.79 & 47.4 & 1647 & 0.00899 & 77 \\
\hline & 20 & 17.66 & 39.6 & 1740 & 0.00488 & 142 \\
\hline & 10 & 18.56 & 33.6 & 1834 & 0.00144 & 481 \\
\hline & 5 & 19.02 & 1.2 & 1901 & 0.000309 & 2,240 \\
\hline & 0 & 19.58 & 34.8 & 1935 & 0 & 0 \\
\hline \multirow[t]{16}{*}{3} & 0 & 5.07 & 4.2 & 504 & 0 & \\
\hline & 5 & 5.63 & 37.8 & 538 & 0.000206 & 3,370 \\
\hline & 10 & 6.09 & 5.4 & 605 & 0.000961 & 721 \\
\hline & 20 & 6.99 & 59.4 & 659 & 0.00325 & 213 \\
\hline & 30 & 7.86 & 51.6 & 752 & 0.006 & 116 \\
\hline & 40 & 8.73 & 43.8 & 844 & 0.00874 & 79.3 \\
\hline & 50 & 9.62 & 37.2 & 937 & 0.0112 & 61.8 \\
\hline & 60 & 10.58 & 34.8 & 1035 & 0.0133 & 52.2 \\
\hline & 70 & 12.17 & 10.2 & 1210 & 0.0148 & 46.8 \\
\hline & 70.09 & 12.32 & 19.2 & 1219 & 0.0148 & 46.8 \\
\hline & 70 & 12.48 & 28.8 & 1229 & 0.0148 & 46.8 \\
\hline & 60 & 14.07 & 4.2 & 1404 & 0.0133 & 52.2 \\
\hline & 50 & 15.03 & 1.8 & 1502 & 0.0112 & 61.8 \\
\hline & 40 & 15.92 & 55.2 & 1555 & 0.00874 & 79.3 \\
\hline & 30 & 16.79 & 47.4 & 1647 & 0.006 & 116 \\
\hline & 20 & 17.66 & 39.6 & 1740 & 0.00325 & 213 \\
\hline
\end{tabular}




\begin{tabular}{|c|c|c|c|c|c|c|}
\hline & 10 & 18.56 & 33.6 & 1834 & 0.000961 & 721 \\
\hline & 5 & 19.02 & 1.2 & 1901 & 0.000206 & 3,370 \\
\hline & 0 & 19.58 & 34.8 & 1935 & 0 & \\
\hline \multirow[t]{19}{*}{4} & 0 & 5.07 & 4.2 & 504 & 0 & 0 \\
\hline & 5 & 5.63 & 37.8 & 538 & 0.000154 & 4,490 \\
\hline & 10 & 6.09 & 5.4 & 605 & 0.00072 & 962 \\
\hline & 20 & 6.99 & 59.4 & 659 & 0.00244 & 284 \\
\hline & 30 & 7.86 & 51.6 & 752 & 0.0045 & 154 \\
\hline & 40 & 8.73 & 43.8 & 844 & 0.00655 & 106 \\
\hline & 50 & 9.62 & 37.2 & 937 & 0.00841 & 82.4 \\
\hline & 60 & 10.58 & 34.8 & 1035 & 0.00995 & 69.6 \\
\hline & 70 & 12.17 & 10.2 & 1210 & 0.0111 & 62.4 \\
\hline & 70.09 & 12.32 & 19.2 & 1219 & 0.0111 & 62.4 \\
\hline & 70 & 12.48 & 28.8 & 1229 & 0.0111 & 62.4 \\
\hline & 60 & 14.07 & 4.2 & 1404 & 0.00995 & 69.6 \\
\hline & 50 & 15.03 & 1.8 & 1502 & 0.00841 & 82.4 \\
\hline & 40 & 15.92 & 55.2 & 1555 & 0.00655 & 106 \\
\hline & 30 & 16.79 & 47.4 & 1647 & 0.0045 & 154 \\
\hline & 20 & 17.66 & 39.6 & 1740 & 0.00244 & 284 \\
\hline & 10 & 18.56 & 33.6 & 1834 & 0.00072 & 962 \\
\hline & 5 & 19.02 & 1.2 & 1901 & 0.000154 & 4,490 \\
\hline & 0 & 19.58 & 34.8 & 1935 & 0 & \\
\hline \multirow[t]{19}{*}{5} & 0 & 5.07 & 4.2 & 504 & 0 & 0 \\
\hline & 5 & 5.63 & 37.8 & 538 & 0.000123 & 5,610 \\
\hline & 10 & 6.09 & 5.4 & 605 & 0.000576 & 1,200 \\
\hline & 20 & 6.99 & 59.4 & 659 & 0.00195 & 355 \\
\hline & 30 & 7.86 & 51.6 & 752 & 0.0036 & 193 \\
\hline & 40 & 8.73 & 43.8 & 844 & 0.00524 & 132 \\
\hline & 50 & 9.62 & 37.2 & 937 & 0.00673 & 103 \\
\hline & 60 & 10.58 & 34.8 & 1035 & 0.00796 & 87 \\
\hline & 70 & 12.17 & 10.2 & 1210 & 0.00888 & 78 \\
\hline & 70.09 & 12.32 & 19.2 & 1219 & 0.00889 & 78 \\
\hline & 70 & 12.48 & 28.8 & 1229 & 0.00888 & 78 \\
\hline & 60 & 14.07 & 4.2 & 1404 & 0.00796 & 87 \\
\hline & 50 & 15.03 & 1.8 & 1502 & 0.00673 & 103 \\
\hline & 40 & 15.92 & 55.2 & 1555 & 0.00524 & 132 \\
\hline & 30 & 16.79 & 47.4 & 1647 & 0.0036 & 193 \\
\hline & 20 & 17.66 & 39.6 & 1740 & 0.00195 & 355 \\
\hline & 10 & 18.56 & 33.6 & 1834 & 0.000576 & 1,200 \\
\hline & 5 & 19.02 & 1.2 & 1901 & 0.000123 & 5,610 \\
\hline & 0 & 19.58 & 34.8 & 1935 & 0 & \\
\hline
\end{tabular}


TABLE S4. Photochemical (sunlight) half-life of $\mathrm{NH}_{2} \mathrm{Cl}$ at latitude $40^{\circ}$, longitude $80^{\circ}$ in water with $44 \mu \mathrm{M}$ DOC as $C\left(0.53 \mathrm{mg} \mathrm{L}^{-1}\right)$ in the winter and summer. Modeled data using GCSOLARS.

\begin{tabular}{|c|c|c|c|c|c|c|}
\hline $\begin{array}{l}\text { Depth } \\
(\mathrm{m})\end{array}$ & Zen. Ang. & $\begin{array}{c}\text { Time } \\
\text { (hours) }\end{array}$ & $\min$ & hours & $\begin{array}{c}\mathrm{k} \\
\left(\mathrm{hr}^{-1}\right)\end{array}$ & $\begin{array}{l}T_{1 / 2} \\
\text { (hr) }\end{array}$ \\
\hline \multicolumn{7}{|c|}{ Winter } \\
\hline \multirow[t]{9}{*}{0} & 0 & 7.54 & 32.4 & 732 & 0 & \\
\hline & 5 & 8.12 & 7.2 & 807 & 0.00142 & 488 \\
\hline & 10 & 8.65 & 39 & 839 & 0.00627 & 111 \\
\hline & 20 & 9.86 & 51.6 & 952 & 0.0217 & 31.9 \\
\hline & 29.86 & 12.42 & 25.2 & 1225 & 0.0396 & 17.5 \\
\hline & 20 & 14.92 & 55.2 & 1455 & 0.0217 & 31.9 \\
\hline & 10 & 16.19 & 11.4 & 1611 & 0.00627 & 111 \\
\hline & 5 & 16.71 & 42.6 & 1643 & 0.00142 & 488 \\
\hline & 0 & 17.29 & 17.4 & 1717 & 0 & \\
\hline \multirow[t]{9}{*}{1} & 0 & 7.54 & 32.4 & 732 & 0 & \\
\hline & 5 & 8.12 & 7.2 & 807 & 0.000718 & 965 \\
\hline & 10 & 8.65 & 39 & 839 & 0.00293 & 237 \\
\hline & 20 & 9.86 & 51.6 & 952 & 0.00948 & 73.1 \\
\hline & 29.86 & 12.42 & 25.2 & 1225 & 0.0171 & 40.6 \\
\hline & 20 & 14.92 & 55.2 & 1455 & 0.00948 & 73.1 \\
\hline & 10 & 16.19 & 11.4 & 1611 & 0.00293 & 237 \\
\hline & 5 & 16.71 & 42.6 & 1643 & 0.000718 & 965 \\
\hline & 0 & 17.29 & 17.4 & 1717 & 0 & \\
\hline \multirow[t]{9}{*}{2} & 0 & 7.54 & 32.4 & 732 & 0 & \\
\hline & 5 & 8.12 & 7.2 & 807 & 0.000437 & 1,580 \\
\hline & 10 & 8.65 & 39 & 839 & 0.00173 & 400 \\
\hline & 20 & 9.86 & 51.6 & 952 & 0.00549 & 126 \\
\hline & 29.86 & 12.42 & 25.2 & 1225 & 0.00987 & 70.2 \\
\hline & 20 & 14.92 & 55.2 & 1455 & 0.00549 & 126 \\
\hline & 10 & 16.19 & 11.4 & 1611 & 0.00173 & 400 \\
\hline & 5 & 16.71 & 42.6 & 1643 & 0.000437 & 1,580 \\
\hline & 0 & 17.29 & 17.4 & 1717 & 0 & \\
\hline \multirow[t]{9}{*}{3} & 0 & 7.54 & 32.4 & 732 & 0 & \\
\hline & 5 & 8.12 & 7.2 & 807 & 0.000304 & 2,280 \\
\hline & 10 & 8.65 & 39 & 839 & 0.00119 & 582 \\
\hline & 20 & 9.86 & 51.6 & 952 & 0.00375 & 185 \\
\hline & 29.86 & 12.42 & 25.2 & 1225 & 0.00674 & 103 \\
\hline & 20 & 14.92 & 55.2 & 1455 & 0.00375 & 185 \\
\hline & 10 & 16.19 & 11.4 & 1611 & 0.00119 & 582 \\
\hline & 5 & 16.71 & 42.6 & 1643 & 0.000304 & 2,280 \\
\hline & 0 & 17.29 & 17.4 & 1717 & 0 & \\
\hline 4 & 0 & 7.54 & 32.4 & 732 & 0 & \\
\hline
\end{tabular}




\begin{tabular}{|c|c|c|c|c|c|c|}
\hline & 5 & 8.12 & 7.2 & 807 & 0.00023 & 3,020 \\
\hline & 10 & 8.65 & 39 & 839 & 0.000899 & 771 \\
\hline & 20 & 9.86 & 51.6 & 952 & 0.00282 & 245 \\
\hline & 29.86 & 12.42 & 25.2 & 1225 & 0.00508 & 137 \\
\hline & 20 & 14.92 & 55.2 & 1455 & 0.00282 & 245 \\
\hline & 10 & 16.19 & 11.4 & 1611 & 0.000899 & 771 \\
\hline & 5 & 16.71 & 42.6 & 1643 & 0.00023 & 3,020 \\
\hline & 0 & 17.29 & 17.4 & 1717 & 0 & \\
\hline 5 & 0 & 7.54 & 32.4 & 732 & 0 & \\
\hline & 5 & 8.12 & 7.2 & 807 & 0.000184 & 3,760 \\
\hline & 10 & 8.65 & 39 & 839 & 0.00072 & 963 \\
\hline & 20 & 9.86 & 51.6 & 952 & 0.00226 & 306 \\
\hline & 29.86 & 12.42 & 25.2 & 1225 & 0.00407 & 170 \\
\hline & 20 & 14.92 & 55.2 & 1455 & 0.00226 & 306 \\
\hline & 10 & 16.19 & 11.4 & 1611 & 0.00072 & 963 \\
\hline & 5 & 16.71 & 42.6 & 1643 & 0.000184 & 3,760 \\
\hline & 0 & 17.29 & 17.4 & 1717 & 0 & \\
\hline $\begin{array}{c}\text { Depth } \\
(\mathrm{m})\end{array}$ & Zen. Ang. & $\begin{array}{c}\text { Time } \\
\text { (hours) }\end{array}$ & $\min$ & hours & $\begin{array}{c}\mathrm{k} \\
\left(\mathrm{hr}^{-1}\right)\end{array}$ & $\begin{array}{l}T_{1 / 2} \\
(\mathrm{hr})\end{array}$ \\
\hline \multicolumn{7}{|c|}{ Summer } \\
\hline 0 & 0 & 5.07 & 4.2 & 504 & 0 & \\
\hline & 5 & 5.63 & 37.8 & 538 & 0.00142 & 488 \\
\hline & 10 & 6.09 & 5.4 & 605 & 0.00627 & 110 \\
\hline & 20 & 6.99 & 59.4 & 659 & 0.0218 & 31.9 \\
\hline & 30 & 7.86 & 51.6 & 752 & 0.04 & 17.3 \\
\hline & 40 & 8.73 & 43.8 & 844 & 0.0567 & 12.2 \\
\hline & 50 & 9.62 & 37.2 & 937 & 0.0703 & 9.86 \\
\hline & 60 & 10.58 & 34.8 & 1035 & 0.0805 & 8.61 \\
\hline & 70 & 12.17 & 10.2 & 1210 & 0.0875 & 7.92 \\
\hline & 70.09 & 12.32 & 19.2 & 1219 & 0.0876 & 7.91 \\
\hline & 70 & 12.48 & 28.8 & 1229 & 0.0875 & 7.92 \\
\hline & 60 & 14.07 & 4.2 & 1404 & 0.0805 & 8.61 \\
\hline & 50 & 15.03 & 1.8 & 1502 & 0.0703 & 9.86 \\
\hline & 40 & 15.92 & 55.2 & 1555 & 0.0567 & 12.2 \\
\hline & 30 & 16.79 & 47.4 & 1647 & 0.04 & 17.3 \\
\hline & 20 & 17.66 & 39.6 & 1740 & 0.0218 & 31.9 \\
\hline & 10 & 18.56 & 33.6 & 1834 & 0.00627 & 110 \\
\hline & 5 & 19.02 & 1.2 & 1901 & 0.00142 & 488 \\
\hline & 0 & 19.58 & 34.8 & 1935 & 0 & \\
\hline \multirow[t]{7}{*}{1} & 0 & 5.07 & 4.2 & 504 & 0 & \\
\hline & 5 & 5.63 & 37.8 & 538 & 0.000719 & 964 \\
\hline & 10 & 6.09 & 5.4 & 605 & 0.00293 & 237 \\
\hline & 20 & 6.99 & 59.4 & 659 & 0.00949 & 73 \\
\hline & 30 & 7.86 & 51.6 & 752 & 0.0172 & 40.3 \\
\hline & 40 & 8.73 & 43.8 & 844 & 0.0247 & 28.1 \\
\hline & 50 & 9.62 & 37.2 & 937 & 0.0311 & 22.3 \\
\hline
\end{tabular}




\begin{tabular}{|c|c|c|c|c|c|c|}
\hline & 60 & 10.58 & 34.8 & 1035 & 0.0363 & 19.1 \\
\hline & 70 & 12.17 & 10.2 & 1210 & 0.04 & 17.3 \\
\hline & 70.09 & 12.32 & 19.2 & 1219 & 0.04 & 17.3 \\
\hline & 70 & 12.48 & 28.8 & 1229 & 0.04 & 17.3 \\
\hline & 60 & 14.07 & 4.2 & 1404 & 0.0363 & 19.1 \\
\hline & 50 & 15.03 & 1.8 & 1502 & 0.0311 & 22.3 \\
\hline & 40 & 15.92 & 55.2 & 1555 & 0.0247 & 28.1 \\
\hline & 30 & 16.79 & 47.4 & 1647 & 0.0172 & 40.3 \\
\hline & 20 & 17.66 & 39.6 & 1740 & 0.00949 & 73 \\
\hline & 10 & 18.56 & 33.6 & 1834 & 0.00293 & 237 \\
\hline & 5 & 19.02 & 1.2 & 1901 & 0.000719 & 964 \\
\hline & 0 & 19.58 & 34.8 & 1935 & 0 & \\
\hline \multirow[t]{19}{*}{2} & 0 & 5.07 & 4.2 & 504 & 0 & \\
\hline & 5 & 5.63 & 37.8 & 538 & 0.000438 & 1,580 \\
\hline & 10 & 6.09 & 5.4 & 605 & 0.00173 & 400 \\
\hline & 20 & 6.99 & 59.4 & 659 & 0.00549 & 126 \\
\hline & 30 & 7.86 & 51.6 & 752 & 0.00994 & 69.7 \\
\hline & 40 & 8.73 & 43.8 & 844 & 0.0143 & 48.4 \\
\hline & 50 & 9.62 & 37.2 & 937 & 0.0182 & 38 \\
\hline & 60 & 10.58 & 34.8 & 1035 & 0.0214 & 32.3 \\
\hline & 70 & 12.17 & 10.2 & 1210 & 0.0238 & 29.1 \\
\hline & 70.09 & 12.32 & 19.2 & 1219 & 0.0238 & 29.1 \\
\hline & 70 & 12.48 & 28.8 & 1229 & 0.0238 & 29.1 \\
\hline & 60 & 14.07 & 4.2 & 1404 & 0.0214 & 32.3 \\
\hline & 50 & 15.03 & 1.8 & 1502 & 0.0182 & 38 \\
\hline & 40 & 15.92 & 55.2 & 1555 & 0.0143 & 48.4 \\
\hline & 30 & 16.79 & 47.4 & 1647 & 0.0994 & 69.7 \\
\hline & 20 & 17.66 & 39.6 & 1740 & 0.00549 & 126 \\
\hline & 10 & 18.56 & 33.6 & 1834 & 0.00173 & 400 \\
\hline & 5 & 19.02 & 1.2 & 1901 & 0.000438 & 1,580 \\
\hline & 0 & 19.58 & 34.8 & 1935 & 0 & \\
\hline \multirow[t]{15}{*}{3} & 0 & 5.07 & 4.2 & 504 & 0 & \\
\hline & 5 & 5.63 & 37.8 & 538 & 0.000304 & 2,280 \\
\hline & 10 & 6.09 & 5.4 & 605 & 0.00119 & 582 \\
\hline & 20 & 6.99 & 59.4 & 659 & 0.00375 & 185 \\
\hline & 30 & 7.86 & 51.6 & 752 & 0.00679 & 102 \\
\hline & 40 & 8.73 & 43.8 & 844 & 0.0098 & 70.7 \\
\hline & 50 & 9.62 & 37.2 & 937 & 0.0125 & 55.4 \\
\hline & 60 & 10.58 & 34.8 & 1035 & 0.0148 & 47 \\
\hline & 70 & 12.17 & 10.2 & 1210 & 0.0164 & 42.2 \\
\hline & 70.09 & 12.32 & 19.2 & 1219 & 0.0164 & 42.2 \\
\hline & 70 & 12.48 & 28.8 & 1229 & 0.0164 & 42.2 \\
\hline & 60 & 14.07 & 4.2 & 1404 & 0.0148 & 47 \\
\hline & 50 & 15.03 & 1.8 & 1502 & 0.0125 & 55.4 \\
\hline & 40 & 15.92 & 55.2 & 1555 & 0.0098 & 70.7 \\
\hline & 30 & 16.79 & 47.4 & 1647 & 0.00679 & 102 \\
\hline
\end{tabular}




\begin{tabular}{|c|c|c|c|c|c|c|}
\hline & 20 & 17.66 & 39.6 & 1740 & 0.00375 & 185 \\
\hline & 10 & 18.56 & 33.6 & 1834 & 0.00119 & 582 \\
\hline & 5 & 19.02 & 1.2 & 1901 & 0.000304 & 2,280 \\
\hline & 0 & 19.58 & 34.8 & 1935 & 0 & \\
\hline \multirow[t]{19}{*}{4} & 0 & 5.07 & 4.2 & 504 & 0 & \\
\hline & 5 & 5.63 & 37.8 & 538 & 0.00023 & 3,010 \\
\hline & 10 & 6.09 & 5.4 & 605 & 0.000899 & 770 \\
\hline & 20 & 6.99 & 59.4 & 659 & 0.00283 & 245 \\
\hline & 30 & 7.86 & 51.6 & 752 & 0.00511 & 136 \\
\hline & 40 & 8.73 & 43.8 & 844 & 0.00739 & 93.7 \\
\hline & 50 & 9.62 & 37.2 & 937 & 0.00945 & 73.4 \\
\hline & 60 & 10.58 & 34.8 & 1035 & 0.0112 & 62.1 \\
\hline & 70 & 12.17 & 10.2 & 1210 & 0.0124 & 55.8 \\
\hline & 70.09 & 12.32 & 19.2 & 1219 & 0.0124 & 55.8 \\
\hline & 70 & 12.48 & 28.8 & 1229 & 0.0124 & 55.8 \\
\hline & 60 & 14.07 & 4.2 & 1404 & 0.0112 & 62.1 \\
\hline & 50 & 15.03 & 1.8 & 1502 & 0.00945 & 73.4 \\
\hline & 40 & 15.92 & 55.2 & 1555 & 0.00739 & 93.7 \\
\hline & 30 & 16.79 & 47.4 & 1647 & 0.00511 & 136 \\
\hline & 20 & 17.66 & 39.6 & 1740 & 0.00283 & 245 \\
\hline & 10 & 18.56 & 33.6 & 1834 & 0.000899 & 770 \\
\hline & 5 & 19.02 & 1.2 & 1901 & 0.00023 & 3.01 \\
\hline & 0 & 19.58 & 34.8 & 1935 & 0 & \\
\hline \multirow[t]{19}{*}{5} & 0 & 5.07 & 4.2 & 504 & 0 & \\
\hline & 5 & 5.63 & 37.8 & 538 & 0.00184 & 3,760 \\
\hline & 10 & 6.09 & 5.4 & 605 & 0.000721 & 962 \\
\hline & 20 & 6.99 & 59.4 & 659 & 0.00226 & 306 \\
\hline & 30 & 7.86 & 51.6 & 752 & 0.00409 & 169 \\
\hline & 40 & 8.73 & 43.8 & 844 & 0.00592 & 117 \\
\hline & 50 & 9.62 & 37.2 & 937 & 0.00757 & 91.6 \\
\hline & 60 & 10.58 & 34.8 & 1035 & 0.00894 & 77.5 \\
\hline & 70 & 12.17 & 10.2 & 1210 & 0.00996 & 69.2 \\
\hline & 70.09 & 12.32 & 19.2 & 1219 & 0.00996 & 69.2 \\
\hline & 70 & 12.48 & 28.8 & 1229 & 0.00996 & 69.2 \\
\hline & 60 & 14.07 & 4.2 & 1404 & 0.00894 & 77.5 \\
\hline & 50 & 15.03 & 1.8 & 1502 & 0.00757 & 91.6 \\
\hline & 40 & 15.92 & 55.2 & 1555 & 0.00592 & 117 \\
\hline & 30 & 16.79 & 47.4 & 1647 & 0.00409 & 169 \\
\hline & 20 & 17.66 & 39.6 & 1740 & 0.00226 & 306 \\
\hline & 10 & 18.56 & 33.6 & 1834 & 0.000721 & 962 \\
\hline & 5 & 19.02 & 1.2 & 1901 & 0.000184 & 3,760 \\
\hline & 0 & 19.58 & 34.8 & 1935 & 0 & \\
\hline
\end{tabular}


TABLE S5. Photochemical (sunlight) half-life of $\mathrm{NH}_{2} \mathrm{Cl}$ at latitude $40^{\circ}$, longitude $80^{\circ}$ in water with $1.47 \mathrm{mM} \mathrm{DOC}$ as $\mathrm{C}\left(17.6 \mathrm{mg} \mathrm{L}^{-1}\right)$ in the winter and summer. Modeled data using GCSOLARS.

\begin{tabular}{|c|c|c|c|c|c|c|}
\hline $\begin{array}{l}\text { Depth } \\
(\mathrm{m})\end{array}$ & Zen. Ang. & $\begin{array}{c}\text { Time } \\
\text { (hours) }\end{array}$ & $\min$ & hours & $\begin{array}{c}\mathrm{k} \\
\left(\mathrm{hr}^{-1}\right)\end{array}$ & $\begin{array}{l}\mathrm{T}_{1 / 2} \\
(\mathrm{hr}) \\
\end{array}$ \\
\hline \multicolumn{7}{|c|}{ Winter } \\
\hline \multirow[t]{9}{*}{0} & 0 & 7.54 & 32.4 & 732 & 0 & \\
\hline & 5 & 8.12 & 7.2 & 807 & 0.00142 & 488 \\
\hline & 10 & 8.65 & 39 & 839 & 0.00626 & 111 \\
\hline & 20 & 9.86 & 51.6 & 952 & 0.0217 & 31.9 \\
\hline & 29.86 & 12.42 & 25.2 & 1225 & 0.0396 & 17.5 \\
\hline & 20 & 14.92 & 55.2 & 1455 & 0.0217 & 31.9 \\
\hline & 10 & 16.19 & 11.4 & 1611 & 0.00626 & 111 \\
\hline & 5 & 16.71 & 42.6 & 1643 & 0.00142 & 488 \\
\hline & 0 & 17.29 & 17.4 & 1717 & 0 & \\
\hline \multirow[t]{9}{*}{1} & 0 & 7.54 & 32.4 & 732 & 0 & \\
\hline & 5 & 8.12 & 7.2 & 807 & 0.0000491 & 14,100 \\
\hline & 10 & 8.65 & 39 & 839 & 0.000173 & 4,010 \\
\hline & 20 & 9.86 & 51.6 & 952 & 0.000518 & 1,340 \\
\hline & 29.86 & 12.42 & 25.2 & 1225 & 0.000921 & 753 \\
\hline & 20 & 14.92 & 55.2 & 1455 & 0.000518 & 1,340 \\
\hline & 10 & 16.19 & 11.4 & 1611 & 0.000173 & 4,010 \\
\hline & 5 & 16.71 & 42.6 & 1643 & 0.0000491 & 14,100 \\
\hline & 0 & 17.29 & 17.4 & 1717 & 0 & \\
\hline \multirow[t]{9}{*}{2} & 0 & 7.54 & 32.4 & 732 & 0 & \\
\hline & 5 & 8.12 & 7.2 & 807 & 0.0000245 & 28,300 \\
\hline & 10 & 8.65 & 39 & 839 & 0.0000863 & 8,030 \\
\hline & 20 & 9.86 & 51.6 & 952 & 0.000259 & 2,670 \\
\hline & 29.86 & 12.42 & 25.2 & 1225 & 0.00046 & 1,510 \\
\hline & 20 & 14.92 & 55.2 & 1455 & 0.000259 & 2,670 \\
\hline & 10 & 16.19 & 11.4 & 1611 & 0.0000863 & 8,030 \\
\hline & 5 & 16.71 & 42.6 & 1643 & 0.0000245 & 28,300 \\
\hline & 0 & 17.29 & 17.4 & 1717 & 0 & \\
\hline $\begin{array}{l}\text { Depth } \\
(\mathrm{m})\end{array}$ & Zen. Ang. & $\begin{array}{c}\text { Time } \\
\text { (hours) }\end{array}$ & $\min$ & hours & $\begin{array}{c}\mathrm{k} \\
\left(\mathrm{hr}^{-1}\right)\end{array}$ & $\begin{array}{l}\mathrm{T}_{1 / 2} \\
\text { (hr) }\end{array}$ \\
\hline \multicolumn{7}{|c|}{ Summer } \\
\hline \multirow[t]{9}{*}{0} & 0 & 5.07 & 4.2 & 504 & 0 & \\
\hline & 5 & 5.63 & 37.8 & 538 & 0.00142 & 488 \\
\hline & 10 & 6.09 & 5.4 & 605 & 0.00627 & 110 \\
\hline & 20 & 6.99 & 59.4 & 659 & 0.0217 & 31.9 \\
\hline & 30 & 7.86 & 51.6 & 752 & 0.0399 & 17.3 \\
\hline & 40 & 8.73 & 43.8 & 844 & 0.0567 & 12.2 \\
\hline & 50 & 9.62 & 37.2 & 937 & 0.0703 & 9.86 \\
\hline & 60 & 10.58 & 34.8 & 1035 & 0.0805 & 8.61 \\
\hline & 70 & 12.17 & 10.2 & 1210 & 0.0875 & 7.92 \\
\hline
\end{tabular}




\begin{tabular}{|c|c|c|c|c|c|c|}
\hline & 70.09 & 12.32 & 19.2 & 1219 & 0.0875 & 7.92 \\
\hline & 70 & 12.48 & 28.8 & 1229 & 0.0875 & 7.92 \\
\hline & 60 & 14.07 & 4.2 & 1404 & 0.0805 & 8.61 \\
\hline & 50 & 15.03 & 1.8 & 1502 & 0.0703 & 9.86 \\
\hline & 40 & 15.92 & 55.2 & 1555 & 0.0567 & 12.2 \\
\hline & 30 & 16.79 & 47.4 & 1647 & 0.0399 & 17.3 \\
\hline & 20 & 17.66 & 39.6 & 1740 & 0.0217 & 31.9 \\
\hline & 10 & 18.56 & 33.6 & 1834 & 0.00627 & 110 \\
\hline & 5 & 19.02 & 1.2 & 1901 & 0.00142 & 488 \\
\hline & 0 & 19.58 & 34.8 & 1935 & 0 & \\
\hline \multirow[t]{19}{*}{1} & 0 & 5.07 & 4.2 & 504 & 0 & \\
\hline & 5 & 5.63 & 37.8 & 538 & 0.0000491 & 14,100 \\
\hline & 10 & 6.09 & 5.4 & 605 & 0.000173 & 4,010 \\
\hline & 20 & 6.99 & 59.4 & 659 & 0.000519 & 1,340 \\
\hline & 30 & 7.86 & 51.6 & 752 & 0.000927 & 747 \\
\hline & 40 & 8.73 & 43.8 & 844 & 0.00133 & 520 \\
\hline & 50 & 9.62 & 37.2 & 937 & 0.0017 & 408 \\
\hline & 60 & 10.58 & 34.8 & 1035 & 0.00201 & 346 \\
\hline & 70 & 12.17 & 10.2 & 1210 & 0.00223 & 310 \\
\hline & 70.09 & 12.32 & 19.2 & 1219 & 0.00223 & 310 \\
\hline & 70 & 12.48 & 28.8 & 1229 & 0.00223 & 310 \\
\hline & 60 & 14.07 & 4.2 & 1404 & 0.00201 & 346 \\
\hline & 50 & 15.03 & 1.8 & 1502 & 0.0017 & 408 \\
\hline & 40 & 15.92 & 55.2 & 1555 & 0.00133 & 520 \\
\hline & 30 & 16.79 & 47.4 & 1647 & 0.000927 & 747 \\
\hline & 20 & 17.66 & 39.6 & 1740 & 0.000519 & 1,340 \\
\hline & 10 & 18.56 & 33.6 & 1834 & 0.000173 & 4,010 \\
\hline & 5 & 19.02 & 1.2 & 1901 & 0.0000491 & 14,100 \\
\hline & 0 & 19.58 & 34.8 & 1935 & 0 & \\
\hline \multirow[t]{17}{*}{2} & 0 & 5.07 & 4.2 & 504 & 0 & \\
\hline & 5 & 5.63 & 37.8 & 538 & 0.0000245 & 28,200 \\
\hline & 10 & 6.09 & 5.4 & 605 & 0.0000864 & 8,020 \\
\hline & 20 & 6.99 & 59.4 & 659 & 0.000259 & 2,670 \\
\hline & 30 & 7.86 & 51.6 & 752 & 0.000464 & 1,490 \\
\hline & 40 & 8.73 & 43.8 & 844 & 0.000667 & 1,040 \\
\hline & 50 & 9.62 & 37.2 & 937 & 0.00085 & 815 \\
\hline & 60 & 10.58 & 34.8 & 1035 & 0.001 & 691 \\
\hline & 70 & 12.17 & 10.2 & 1210 & 0.00112 & 621 \\
\hline & 70.09 & 12.32 & 19.2 & 1219 & 0.00112 & 620 \\
\hline & 70 & 12.48 & 28.8 & 1229 & 0.00112 & 621 \\
\hline & 60 & 14.07 & 4.2 & 1404 & 0.001 & 691 \\
\hline & 50 & 15.03 & 1.8 & 1502 & 0.00085 & 815 \\
\hline & 40 & 15.92 & 55.2 & 1555 & 0.000667 & 1,040 \\
\hline & 30 & 16.79 & 47.4 & 1647 & 0.000464 & 1,490 \\
\hline & 20 & 17.66 & 39.6 & 1740 & 0.000259 & 2,670 \\
\hline & 10 & 18.56 & 33.6 & 1834 & 0.0000864 & 8,020 \\
\hline
\end{tabular}




\begin{tabular}{|r|r|r|r|r|r|r|}
\hline & 5 & 19.02 & 1.2 & 1901 & 0.0000245 & 28,200 \\
\hline & 0 & 19.58 & 34.8 & 1935 & 0 & \\
\hline
\end{tabular}

